\title{
A DATABASE FOR SUPERCONDUCTING CAVITIES FOR THE TESLA TEST FACILITY
}

\author{
P.D. Gall*, A. Goessel, V. Gubarev, J. Iversen, DESY, Notkestr. 85, 22603 Hamburg, Germany
}

\section{Abstract}

We look back on 10 years experience using a database for superconducting cavities for the TESLA Test Facility (TTF). The database was developed to collect data of every preparation step and measurement in order to optimize cavity production and preparation techniques to meet the ambitious goal of high accelerating gradients at high quality factors.

Data from 110 superconducting 9-cell cavities, 50 single cell cavities, several 2 - to 7 -cell cavities and about $60 \mathrm{RF}$ couplers were collected in the database. In addition, company measurements on sub-assemblies and parts forming the next 30 9-cell cavities were stored, thus establishing the database as part of a Quality Management System.

This database is dynamically accessible via an extensive graphical WEB interface based on ORACLE products, which enables the users to select and an alyse the collected data easily from anywhere.

\section{INTRODUCTION}

Reliable production and preparation of superconducting cavities with anticipated high accelerating gradients at high quality factors is needed for any future accelerator applying the "cold" technology. This calls for an effective Quality Management System. Therefore, 10 years ago at DESY a relational database was developed to collect data from every preparation step and measurement result of these superconducting cavities.

Up to now, data from 110 superconducting 9 cell 1.3 $\mathrm{GHz}$ cavities, produced by different European companies and tested at the TESLA Test Facility (TTF) at DESY [1], were collected in this database In addition, the results of manufacturer measurements on sub-assemblies and parts forming the next 30 9-cell cavities were stored and compared with the nominal values using given tolerances. This establishes the database as an effective part of a Quality Management System for the whole cavity production and preparation process at a very detailed level.

In an $R \& D$ program to optimize the production and preparation techniques for the cavities, 50 single cell and several 2- to 7-cell superconducting cavities, partially produced at DESY, have been prepared and tested. Data about these cavities were stored in the database.

The database is dynamically accessible from everywhere via an extensive graphical WEB interface based on ORACLE products: http://tesla.desy.de, choose "Technical" and then "Cavity information". At Jefferson Lab a similar WEB-based system [2] has been developed to provide process control and documentation for SNS cryomodule production.

dieter.gall@desy.de

\section{DATABASE STRUCTURE}

The data are loaded into more than 100 tables, the basic structure of a relational database, representing for each cavity and coupler the essential checks, treatments and measurements [3]:

- Essential properties of the cavity material;

- Production data provided by the manufacturers of the cavities and by the acceptance control at DESY;

- Results from cavity eccentricity and frequency tuning (field flatness) measurements;

- Temperatures, pressures and mass spectra for the heat treated cavities;

- Parameters and results from degreasing, buffered chemical polishing (BCP) or electro polishing (EP), and high pressure water rinsing (HPR) of the cavities;

- Information about the cavity assembly to vertical and/or horizontal test stands;

- Temperature behaviours and vacuum mass spectra from cooling down the cavities to $2 \mathrm{~K}$;

- $\quad$ RRR measurement results;

- Results from the cavity RF tests under cw and/or pulsed conditions at $2 \mathrm{~K}$;

- Data about testing and conditioning of input couplers with and without cavity;

- Information about assembling a string of 8 9-cell cavities to a module and finally the module to the TTF Linac.

One or more tables ae used to describe these different cavity data. Most tables are related to a specific cavity by the cavity name and a timestamp for its treatment or measurement. We use the ORACLE Relational Data Base Management System (RDBMS) which is accessible via a Graphical User Interface (GUI) through the WEB. ORACLE uses SQL, the Structured Query Language, which allows complex queries and table links.

\section{DATA COLLECTION}

Big data files from cavity tests and preparation steps are automatically generated by control software and loaded directly into the database.

Other data, like the measurements from the entrance control at DESY, information about input coupler assembly and so on, are collected manually via GUI tools.

Some years ago a commercially available Engineering Data Management System (EDMS) was introduced at DESY. This tool is mainly used to document the mechanical production of the superconducting cavities 
from niobium sheets as well as the subsequent surface conditioning. Measurement results are attached to the EDMS documents as MS EXCEL-, PDF- and ASCIIfiles. We developed a special tool based on EDMS JAVA API to extract, parse and load these data automatically from EDMS to the database (Fig. 1) to make them available for statistical analyses.

The most important data are updated within a day, whereas other data - like the timetables of the processed cavities and the availability of important preparation and measurement devices - are normally updated only once a week.

\begin{tabular}{|c|c|c|c|c|c|}
\hline \multicolumn{6}{|l|}{ 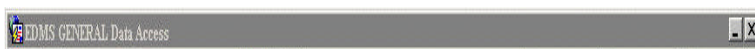 } \\
\hline S 15 & 国国 & 11:21:09 & Dataloal & Prefernices & Quit \\
\hline DMMS Docurents & \multicolumn{5}{|c|}{ 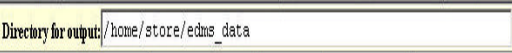 } \\
\hline \multirow{2}{*}{ 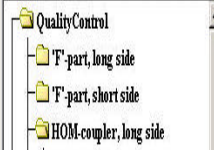 } & \multicolumn{2}{|c|}{ 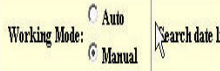 } & \multicolumn{2}{|c|}{ 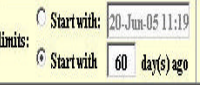 } & 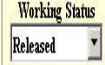 \\
\hline & Tipe & Doculuertit & Lastmodififed & Staths & Int Piff Xls \\
\hline 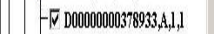 & carthos & $000000000379993, \mathrm{~A}_{1}, 1,1$ & 02-Jun-2005 15:41:16 & LcsReleassed & ]」」 \\
\hline 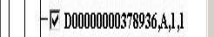 & $\operatorname{carrina}||_{0}^{2}$ & $0000000003789936,1,1,1$ & 02-Jun-2005 15:41:39 & LisisReleased & d]」 \\
\hline 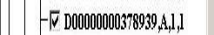 & carina: $\mid$ D. & $0000000000376999,1,1,1$ & 02--Vun-2005 15:41:47| & Liskelessed & I」 $\lrcorner$ \\
\hline 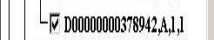 & cariou Do & $0000000000376942,1,1,1$ & 02-Jun-2005 15:41:54 & LessReleased & 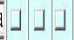 \\
\hline -HOM-eouler, slurtside & & & & & $\mathbb{Q} \mathbb{Q}$ \\
\hline - - Doupler hovising, louy side & & & & & $\mathbb{Q} \mathbb{Q}$ \\
\hline - coupler hovingy, short sile & & & & & $\mathbb{Q} \mathbb{Q}$ \\
\hline - Duwb-hell (after trimuing / thegu & & & & & $\mathbb{Q} \mathbb{\mathbb { Q }}$ \\
\hline 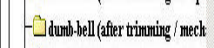 & & & & & $\mathbb{Q} \mathbb{\mathbb { Z }}$ \\
\hline 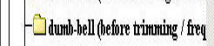 & & & & & $\mathbb{Q} \mathbb{\mathbb { Q }}$ \\
\hline 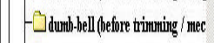 & & & & & $\mathbb{E} \mathbb{\mathbb { Q }}$ \\
\hline - - general & & & & & $\mathbb{\mathbb { Q }} \mathbb{\mathbb { Q }}$ \\
\hline 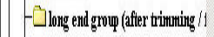 & & & & & $\mathbb{Q} \mathbb{\mathbb { B }}$ \\
\hline 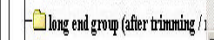 & & & & & $\mathbb{Q} \mathbb{Q}$ \\
\hline & & & & & $\mathbb{Q} \mathbb{Q} \mathbb{Q}$ \\
\hline 4 & & & & & $\mathbb{Q} \mathbb{\mathbb { Q }}$ \\
\hline
\end{tabular}

Figure 1: Data loading via EXCEL Forms from EDMS.

\section{DATA HANDLING}

\section{Views}

For an easier access to the data stored in the database we created Views which are logical tables combining several physical tables of the database. Some Views connect data from tables corresponding to one cavity treatment or measurement type, e.g. the View gathering information about the RF tests under $\mathrm{cw}$ conditions (3 tables involved). The complex Summary Views combine data from tables describing different cavity preparation and/or measurement procedures, e.g. the View Last_Test_Results combining data about the preparation and RF measurement history of a cavity (more than 10 different preparations and measurements from 20 tables). The Views facilitate direct access to complex data queries which may be handled by native SQL scripts or by client programs like MS EXCEL using ODBC (Open Data Base Connectivity).

\section{Graphical User Interface}

A Graphical User Interface (GUI) to the database for superconducting cavities for TTF has been implemented [4] on the WEB, based on ORACLE Developer 6i FORMS and GRAPHICS. In consultation with the users, it has been expanded over the years in order to provide a user-friendly way for viewing and analysing the accumulated data (Fig. 2). Since the decision for the "cold" technology for future accelerat ors the number of accesses to our WEB pages has increased dramatically.

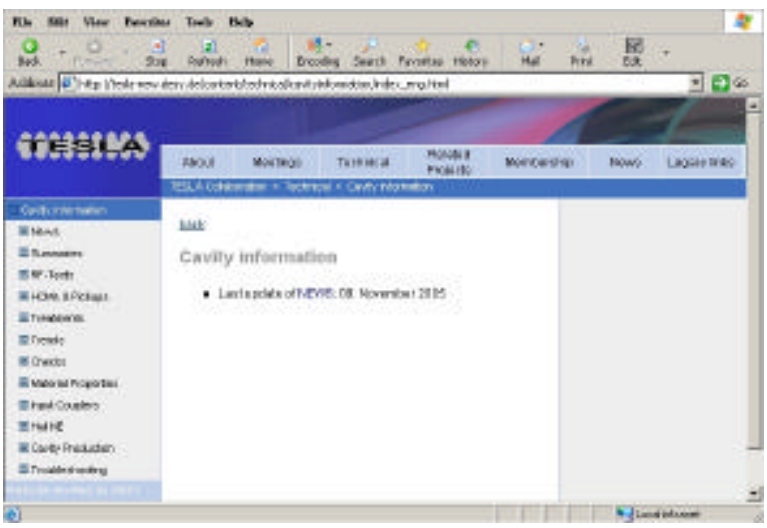

Figure 2: Start page of the GUI to the database for the TTF cavities.

The GUI is based on more than 100 Views. As an example, Fig. 3 shows the WEB page RF-Test Results, which guides the user to records of RF measurements retrieved from the dat abase under specific view points.
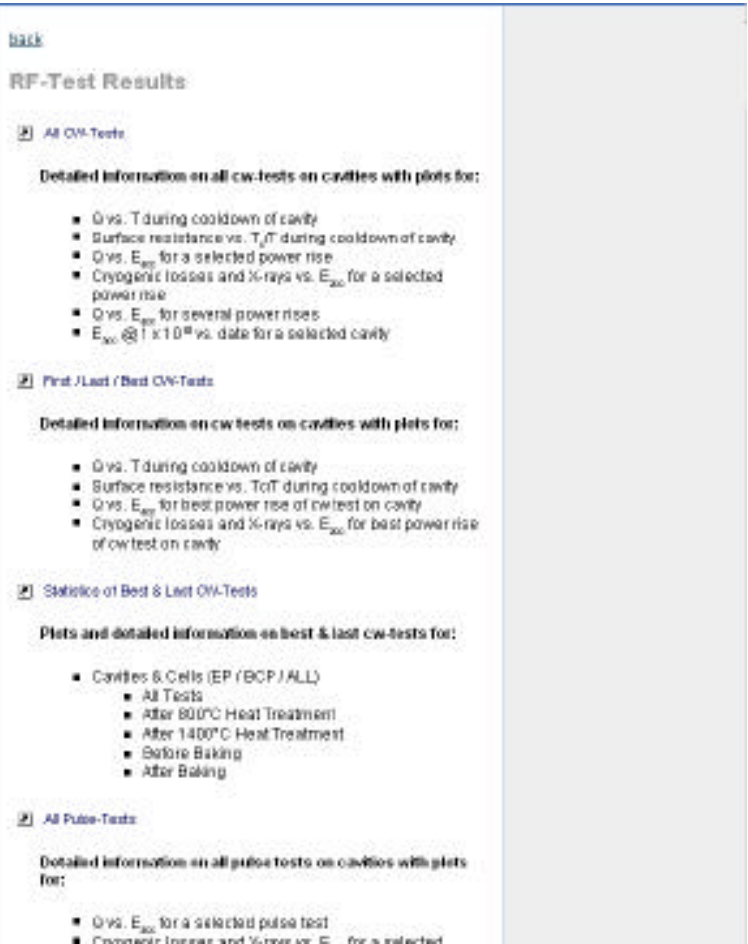
Figure 3: Example of aWEB page for specific queries about RF measurements.

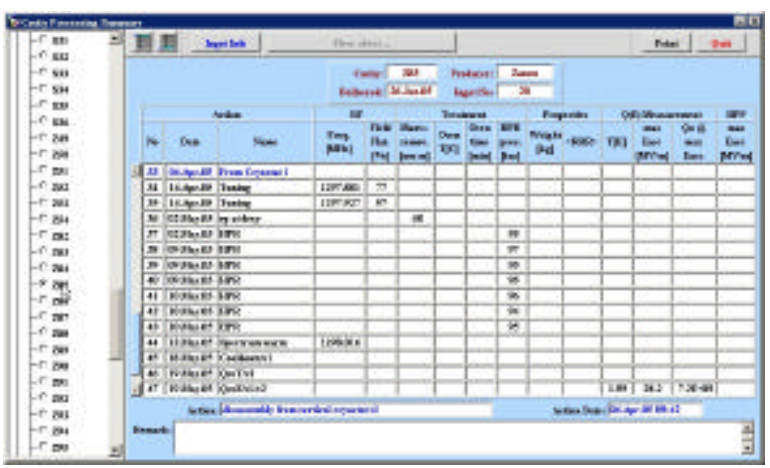

Figure 4: Example for chronological applications: The Summary Information on preparations and tests performed on a selected cavity.

The GUI includes about 50 chronological (Fig. 4), 50 statistical (Fig. 5) and ca. 20 detailed applications (Fig. 6), where the data are represented either in FORMS where the results of just one cavity can be selected from a list of cavities (Fig. 4), or in multi-frame FORMS where the data of a selected group of cavities may be compared (Fig. 5).

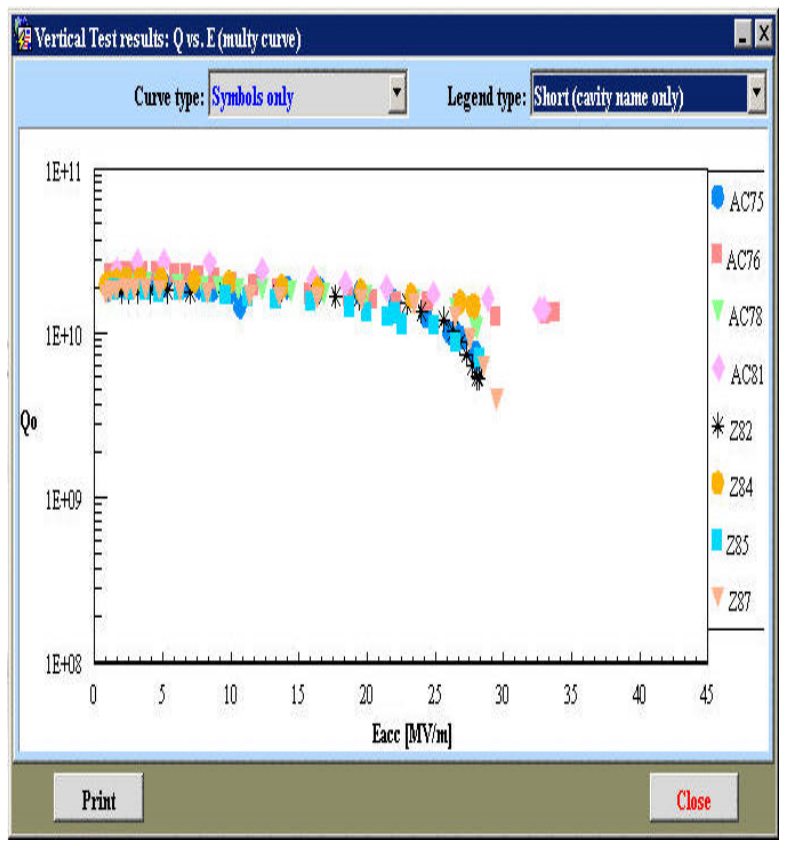

Figure 5: Example for statistical applications: Excitation curves of a selected group of cavities.

On most of the FORMS the use of the right mouse button offers the possibility to select and/or order the retrieved records.

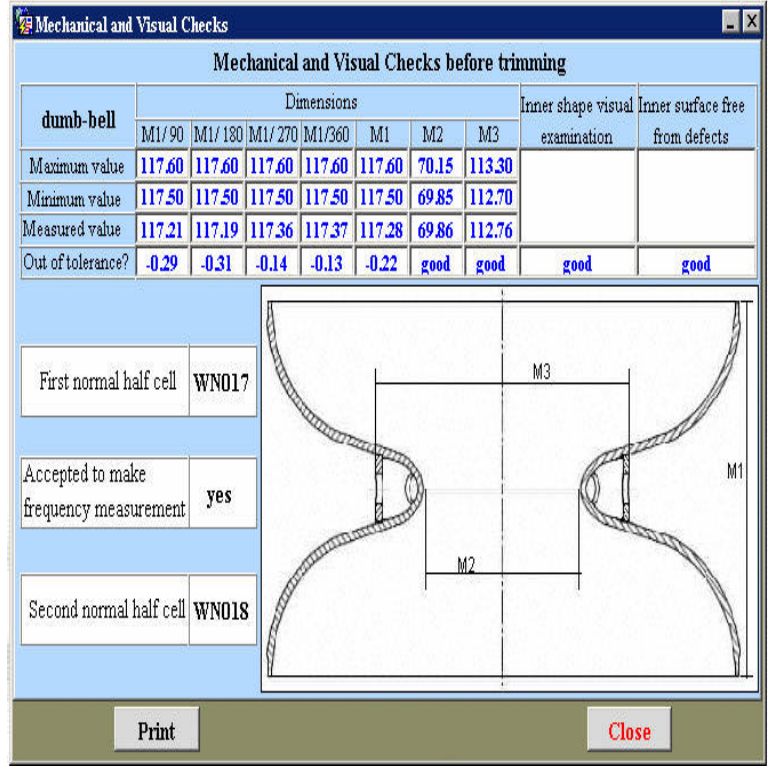

Figure 6 Example for detailed applications: Mechanical and visual checks at a company on a dumb bell, a sub-unit of a cavity.

\section{Report Generation System}

In order to obtain hardcopies of the results provided by the GUI, a Report Generation System was developed. It is based on ORACLE REPORTS and GRAPHICS and produces Table, Graphical or Informational (combined) Reports. Alternatively, the RGS can generate Postcript, HTML, XML, PDF or TEXT (ASCII) files .

\section{REFERENCES}

[1] D.A. Edwards, TESLA Test Facility Linac - Design Report, TESLA 95-1, 1995.

[2] B. Aune et al., "Superconducting TESLA Cavities", Phys. Rev. ST Accel. Beam 3, 092001, 2000.

[3] J. Preble et al., "Process Control and Documentation for SNS Cryomodule Production", Poster TuP32 of the $11^{\text {th }}$ Workshop on RF Superconductivity, Luebeck/Travemuende, Germany, 2003.

[4] P.D. Gall et al., "A Database for Superconducting Cavities for the TESLA Test Facility", Proceedings of the $11^{\text {th }}$ Workshop on RF Superconductivity, Luebeck/Travemuende, Germany, 2003. 